\title{
CRITICAL PHENOMENA IN 2D ANISOTROPIC SPIN SYSTEMS WITH WEAK DISORDER
}

\author{
G. Jug ${ }^{\dagger}$, B. N. Shalaev ${ }^{\dagger \dagger}$ \\ ${ }^{\dagger}$ INFM and Instituto di Scienze Matematiche, Fisiche e Chimiche \\ Università di Milano a Como, Via Lucini 3, 22100 Como (Italy) \\ ${ }^{\dagger}$ A. F. Ioffe Physical \&; Technical Institute, Russian Academy of Sciences \\ 194021 St.Petersburg (Russia) \\ (Received April 4, 1998)
}

\begin{abstract}
The critical behavior of 2D anisotropic systems with weak quenched disorder described by the so-called generalized Ashkin-Teller model (GATM), including the Ising model with random bonds, the dilute Baxter model, the impure $N$-color Ashkin-Teller model, and minimal conformal field theory models (MCFTM) with $c<1$ ( $c$ is the central charge) perturbed by randomness is discussed. All these models except MCFTM were found to belong to the Ising model universality class. Critical exponents of disordered MCFTM are calculated within perturbative expansions in the powers of $\epsilon=c-1 / 2$. RG flows exhibit the rounding of fluctuation-driven first-order phase transitions by disorder.
\end{abstract}

Key words: critical phenomena, 2D anisotropic spin systems, Ashkin-Teller model, weak quenched disorder.

PACS number(s): 68.35.Rh, 75.10.Hk, 82.65.Dp

The physics of disorder continues to be a very active and important area of research, particularly for two-dimensional (2D) condensed matter systems where a rich scenario of homogeneous-system phase transitions is known to occur [1]. These systems encompass a large spectrum of important condensed-matter structures ranging from physisorbed layers to surfaces, from layered structures to vortex lattices. The effects of disorder on the phase transitions in these cooperative systems is currently subject of intense investigations through both theory and experiments.

The case of quenched impurities in layered magnetic and superconducting structures represents a wellestablished and long-studied configuration of defects. However, for physisorbed layers, thin films and crystal surfaces quenched disorder represents still somewhat of a novel playground for both predictions and experiments. Normally, impurities appear with thermally annealed, equilibrium distributions in these systems and the situation where heavy or chemisorbed impurities are introduced in a controlled fashion is still awaiting systematic investigation. We will argue, however, in this Letter that interesting new aspects are ready to be studied, and predictions to be verified, for these systems in the presence of quenched disorder.

Besides the recently-debated issues of the effects of disorder on realizations of the $3-$ and 4-state Potts models in physisorbed layers [2], where evidence for symmetry-independent critical behaviour of the random Ising model (RIM) type is emerging, we also recall the question of the suppression of a first-order transition [3] as induced by disorder. Furthermore, a few 2D systems are known to exhibit the remarkable phenomenon of weak universality (inherent to the Baxter or eightvertex model [4]). Examples are the $p(2 \times 1)$ and $p(2 \times 2)$ structures in physisorbed layers [5], direct realizations of the Baxter model, and more recently the fcc(110) noblemetal and fcc(111) rare-gas crystal faces, characterised by the preroughening transition [6]. It is therefore interesting and important to investigate the robustness of the Baxter line to the introduction of inevitable weak quenched disorder.

In this paper we provide some sound theoretical evidence for the RIM superuniversality for a class of discrete-symmetry continuous phase transitions in $2 \mathrm{D}$ systems where disorder leads to the fixed point and critical exponents of the RIM universality class. We verify this rigorously for the $N$-colour and generalised AshkinTeller models, known to correspond in $2 \mathrm{D}$ to a number of experimental realizations [1]. For the minimal conformal-field-theory models (MCFTM) (hence for the 3 - and 4-state Potts models), however, we find that RIM superuniversality holds only approximately, but remarkably to a very high degree of accuracy, when disorder is introduced. Furthermore, we confirm that disorder destroys the Baxter line of variable critical exponents (including that of preroughening) with a full crossover to the RIM behaviour. Some of these features were already obtained some time ago by Dotsenko and Dotsenko [7] (see, however, also Ref. [8]).

We consider, to begin with, the $N$-colour AshkinTeller model (ATM) [9] described by the following Landau effective Hamiltonian, in the presence of weak disorder $\lambda(x)$

$$
\begin{aligned}
H & =\int d^{2} x\left\{\frac{1}{2}\left(\partial_{\mu} \Phi\right)^{2}+\frac{1}{2}\left(m_{0}^{2}+i \lambda(x)\right) \Phi^{2}\right. \\
& \left.+\frac{1}{8} v_{0} \sum_{a=1}^{N} \Phi_{a}^{4}+\frac{1}{8} u_{0}\left(\Phi^{2}\right)^{2}\right\}
\end{aligned}
$$


with $\Phi^{2}=\sum_{a=1}^{N} \Phi_{a}^{2}$ and $\left(\partial_{\mu} \Phi\right)^{2}=\sum_{a=1}^{N}\left(\partial_{\mu} \Phi_{a}\right)^{2}$. Here, $\Phi$ is an $N$-component order parameter field, $v_{0}$ and $u_{0} \sim J_{4}$ are suitable couplings, $m_{0}^{2} \sim \tau=\left(T-T_{c}\right) / T_{c}$, with $T_{c}$ the pure system's critical temperature, and $\lambda(x)$ is a Gaussian distributed field taking disorder into account. The homogeneous part of Eq. (1) is the usual Landau expansion for a stack of $N$ Ising systems, coupled to each other by the energy operator:

$$
H_{N A T}=-\sum_{<n n>}\left\{J \sum_{a=1}^{N} s_{i}^{a} s_{j}^{a}+J_{4}\left(\sum_{a=1}^{N} s_{i}^{a} s_{j}^{a}\right)^{2}\right\},
$$

which in the case of $N=2$ defines the eight-vertex or Baxter model [4]. For general $N$, this model describes a number of magnetic and structural transitions in a variety of solids [10], whilst for $N=2$ and in $2 \mathrm{D}$ it describes the weak universality of phase transitions in physisorbed layers and surfaces [5,6]. In the $N=0$ limit, and $\lambda(x)=0$, it represents the Landau expansion for the RIM [11]. Finally, for $N \rightarrow \infty$ Aharony [12] showed that the $\lambda(x)=0$ Hamiltonian is appropriate for the description of the annealed RIM critical behaviour. In 2D the ATM can be studied by means of the fermionization method [13], through which the effective action becomes that of the $O(N)$-symmetric Gross-Neveu model (GNM), with the inclusion of disorder:

$$
\begin{aligned}
S[\bar{\psi}, \psi] & =\int d^{2} x\left[i \bar{\psi}_{a} \hat{\partial} \psi_{a}\right. \\
& \left.+\left(m_{0}^{2}+i \lambda(x)\right) \bar{\psi}_{a} \psi_{a}+u_{0}\left(\bar{\psi}_{a} \psi_{a}\right)^{2}\right]
\end{aligned}
$$

Now $\psi$ is an $N$-component (real) Majorana field; thus, for $N \rightarrow 0$ (and $\lambda(x)=0$ ) the fermionic mapping for the RIM is reproduced. In the absence of $\lambda(x)$ (hence for the pure ATM or, for $N=0$ or $N=\infty$, for the RIM), the behaviour near $T_{c}$ is known [8] from the solution of the one-loop renormalization group (RG) equations. For the thermodynamic functions (correlation length $\xi$ and specific heat $C$ ) we have: $\xi \sim \tau^{-1}|\ln \tau|^{(N-1) /(N-2)}$ and $C \sim|\ln \tau|^{N /(2-N)}$, while for the correlation-function at criticality $G(R)$, susceptibility $\chi$ and magnetization $M$ we get: $G(R) \sim R^{-1 / 4}, \chi \sim \tau^{-7 / 4}|\ln \tau|^{7(N-1) / 4(N-2)}$ and $M \sim(-\tau)^{1 / 8}|\ln (-\tau)|^{(N-1) / 8(N-2)}$. These results clearly show how the Baxter model, $N=2$, is a special case within the RG scheme and needs a separate solution, via, e.g., bosonization or the mapping on the massive Thirring model, leading to continuously-varying exponents.

Let us now discuss the effects of disorder on the ATM, $\lambda(x) \neq 0$ and finite $N$. It is convenient to slightly generalize the cubic Hamiltonian of Eq. (1), by introducing two interacting $N$ - and $M$-colour ATMs

$$
\begin{aligned}
H & =\int d^{2} x\left\{\frac{1}{2}\left(\partial_{\mu} \Phi\right)^{2}+\frac{1}{2}\left[m_{0}^{2}+\lambda_{1}(x)\right] \Phi_{a}^{2}+\frac{1}{2}\left[m_{0}^{2}+\lambda_{2}(x)\right] \Phi_{c}^{2}+\frac{1}{8} u_{1}\left(\Phi_{a}^{2}\right)^{2}+\frac{1}{8} u_{2}\left(\Phi_{c}^{2}\right)^{2}\right. \\
& \left.+\frac{1}{8} w_{0} \Phi_{a}^{2} \Phi_{c}^{2}+\frac{1}{8} v_{1} \sum_{a=1}^{N} \Phi_{a}^{4}+\frac{1}{8} v_{2} \sum_{c=N+1}^{N+M} \Phi_{c}^{4}\right\}
\end{aligned}
$$

where $\Phi_{k}, k=1,2, \ldots, M+N$ is an $(M+N)$-component order parameter, $a=1,2, \ldots, N, c=N+1, N+2, \ldots, N+M$, $m_{0}^{2} \sim \tau$ and $v_{\mu}, u_{\mu}>0(\mu, \nu=1,2)$. Summation over repeated indices is understood in all quadratic operators. The Gaussian-distributed disorder fields $\lambda_{\mu}(x)$ may be taken as uncorrelated, $\left\langle\lambda_{\mu}(x) \lambda_{\nu}(y)\right\rangle=z_{\mu} \delta_{\mu \nu} \delta(x-y)$, or correlated, $\left\langle\lambda_{\mu}(x) \lambda_{\nu}(y)\right\rangle=z_{0} \delta(x-y)$. In $4-\epsilon$ dimensions, with and without disorder, this generalized Ashkin-Teller model (GATM) was studied by a number of workers [14] for describing structural and magnetic phase transitions in solids. By applying the standard fermionization and replica tricks, we are lead to an effective action containing a number of quartic terms:

$$
\begin{aligned}
H & =\int d^{2} x\left\{i \bar{\Psi}_{k}^{\alpha} \hat{\partial} \Psi_{k}^{\alpha}+m_{0}^{2} \bar{\Psi}_{k}^{\alpha} \Psi_{k}^{\alpha}+u_{1} \bar{\Psi}_{a}^{\alpha} \Psi_{a}^{\alpha} \bar{\Psi}_{b}^{\alpha} \Psi_{b}^{\alpha}+u_{2} \bar{\Psi}_{c}^{\alpha} \Psi_{c}^{\alpha} \bar{\Psi}_{d}^{\alpha} \Psi_{d}^{\alpha}+w_{0} \bar{\Psi}_{a}^{\alpha} \Psi_{a}^{\alpha} \bar{\Psi}_{c}^{\alpha} \Psi_{c}^{\alpha}\right. \\
& \left.+z_{1} \bar{\Psi}_{a}^{\alpha} \Psi_{a}^{\alpha} \overline{\Psi_{b}^{\beta}} \Psi_{b}^{\beta}+z_{2} \bar{\Psi}_{c}^{\alpha} \Psi_{c}^{\alpha} \bar{\Psi}_{d}^{\beta} \Psi_{d}^{\beta}+r_{0} \bar{\Psi}_{a}^{\alpha} \Psi_{a}^{\alpha} \bar{\Psi}_{c}^{\beta} \Psi_{c}^{\beta}\right\}
\end{aligned}
$$

with $\Psi_{k}^{\alpha}$ the (real) Majorana field, $\alpha, \beta=1,2, \ldots, n(n \rightarrow 0)$ the replica indices, $a, b=1,2, \ldots, N$ and $c, d=N+1, N+$ $2, \ldots, N+M$. Notice the presence of the additional coupling $r_{0}$ (absent in the bare action) induced by renormalization and ensuring the closedness of the operator algebra. We shall see that the presence of the corresponding operator will violate the Harris criterion for the positiveness of the specific heat exponent. The one-loop RG equations read (for $n=0)$ : 


$$
\begin{aligned}
& \frac{d u_{1}}{d t}=-(N-2) u_{1}^{2}-2 z_{1} u_{1}-M w^{2} \\
& \frac{d u_{2}}{d t}=-(M-2) u_{2}^{2}-2 z_{2} u_{2}-N w^{2} \\
& \frac{d w}{d t}=-w\left[(N-1) u_{1}+(M-1) u_{2}+z_{1}+z_{2}\right] \\
& \frac{d z_{1}}{d t}=-2 z_{1}\left[z_{1}+(N-1) u_{1}+2 M r\right] \\
& \frac{d z_{2}}{d t}=-2 z_{2}\left[z_{2}+(M-1) u_{2}+2 N r\right] \\
& \frac{d r}{d t}=-r\left[(N-1) u_{1}+(M-1) u_{2}+z_{1}+z_{2}\right]-w\left[N z_{1}+M z_{2}\right]
\end{aligned}
$$

with the initial conditions $z_{1}(0)=z_{2}(0)=z_{0}, r(0)=0$, for uncorrelated disorder, and $z_{1}(0)=z_{2}(0)=2 r(0)=$ $z_{0}$ for the correlated case. We can now see that for $N=M=1$ (random Baxter, or 2-colour ATM) one arrives at the RG equations first obtained by Dotsenko and Dotsenko [7]. In this case the couplings $u_{1}, u_{2}$ decouple from the others. Also, we have only one coupling constant $z_{1}=z_{2} \equiv z$ and we see that disorder changes the critical behaviour of the 2-colour ATM, characterised by weak universality, to that of the RIM modified by some logarithmic corrections (see also [8] for correlation function asymptotics). Even though the critical exponent $\alpha$ is negative for $w_{0}<0$ (case of preroughening [6]), for uncorrelated disorder we still find a change to RIM critical behaviour due to the presence of the coupling $r$. For correlated disorder and $w_{0}<0$ weak universality persists [7], except for the correlation-function related quantities [8], for which RIM exponents are recovered.

We now come to discuss the effect of disorder on the MCFT models, our main new result. In accordance with the Harris criterion, weak disorder is expected to be relevant in these models since $\alpha=2(m-3) / 3(m-1)$, with $m=3,4, \ldots$, is always positive. In particular, for the 3 -state $(m=5)$ and 4 -state $(m=\infty)$ Potts models we have $\alpha=\frac{1}{3}$ and $\alpha=\frac{2}{3}$, respectively. In some pioneering work, Ludwig [15] and Dotsenko, Picco and Pujol [16] have succeeded in developing a powerful approach, connected to the formalism developed for the GATM, by exploiting a special kind of $\epsilon$-expansion, with $\epsilon=c-\frac{1}{2}(c<1$ being the central charge of the MCFTM and $\frac{1}{2}$ that of the $2 \mathrm{D}$ Ising model). The main result is that the $\beta(u)$ and $\gamma_{\bar{\psi} \psi}(u)$ functions coincide with those of the $O(N)$-symmetric GNM obtained in the minimalsubtraction scheme using dimensional regularization, except for the first trivial term in the $\beta$-function. Although 5 -loop expansions are available, owing to some unknown higher-order coefficients we report the results to $3-$ loop order $[16,17]$

$$
\beta(u)=2 \epsilon u-2(N-2) u^{2}+4(N-2) u^{3}
$$

$$
\begin{gathered}
+2(N-2)(N-7) u^{4}, \\
\gamma_{\bar{\psi} \psi}(u)=2(N-1) u \\
\quad-2(N-1) u^{2}-2(N-1)(2 N-3) u^{3}, \\
\epsilon=(3 m) / 2 m, \quad m=3,4, \ldots
\end{gathered}
$$

From these equations, it follows that the correlation length critical exponent is given by

$$
\begin{aligned}
& \frac{1}{\nu}=\frac{1}{\nu_{0}}+2(N-1)\left[\frac{\epsilon}{N-2}+\frac{\epsilon^{2}}{(N-2)^{2}}-\frac{N \epsilon^{3}}{(N-2)^{3}}\right] \\
& \nu_{0}=\frac{2 m}{3(m-1)}
\end{aligned}
$$

where $\nu_{0}$ refers to the pure system. To check the consistency of this result, we consider the $N \rightarrow \infty$ limit describing annealed disorder, to get $\nu=2 m /(m+3)=\nu_{0} /(1-$ $\left.\alpha_{0}\right)$, in agreement with Fisher renormalization [18]. From [16] it also follows that the $\eta$ exponent remains unchanged in this limit, $\eta=\eta_{0}=(m+3)(m-1) / 4 m(m+1)$ with $q=4 \cos ^{2}(\pi /(m+1))$ for the number of Potts degrees of freedom. For $N=1$ one gets the critical exponents of the pure system.

The critical exponent $\nu_{r}$ for the random models can be obtained by setting $N=0$ in Eq. (7). The results of the apparently convergent resulting series are reported in Table I. One can see that the correlation length exponent is to a very good approximation the same as for the RIM, $\nu_{r} \approx 1$, as was indeed observed in some numerical studies [2]. This is also observed for the exponent $\eta_{r}$ [16], leading to an (approximate) superuniversality of all the MCFT models. It is known, however, that the RIM 
fixed point does not correspond exactly to that for the disordered 4-state Potts model [15,16].

\begin{tabular}{rccccccc}
\hline \hline model & $m$ & $\epsilon$ & $\nu_{0}$ & $\nu_{1}$ & $\nu_{2}$ & $\nu_{3}$ & $\nu_{r}$ \\
\hline TIM & 4 & -0.125 & 0.889 & 0.099 & 0.017 & 0.003 & 1.008 \\
3-PM & 5 & -0.2 & 0.833 & 0.139 & 0.038 & 0.008 & 1.018 \\
TPM & 6 & -0.25 & 0.8 & 0.16 & 0.052 & 0.014 & 1.026 \\
4 -PM & $\infty$ & -0.5 & 0.667 & 0.222 & 0.13 & 0.062 & 1.081 \\
\hline \hline
\end{tabular}

Table I. Critical correlation length exponent $\nu$ for random minimal models: TIM (Tricritical Ising Model), 3-PM (3-state Potts Model), TPM (Tricritical Potts Model), and 4-PM (4-state Potts Model). $m$ denotes the minimal model, $\epsilon=(3-m) / 2 m, \nu_{0}$ is the homogeneous exponent, $\nu_{r}=\nu_{0}+\nu_{1}+\nu_{2}+\nu_{3}$ the random one and $\nu_{n}$ denotes the $n$-loop contribution to $\nu_{r}$.
In conclusion, we have shown the RIM to be a central theme in the physics of disordered 2D discretesymmetry systems. Many models, notably the ATM and GATM, give way to the RIM fixed point in the presence of weak disorder, whilst for others, e.g. the MCFTM, the RIM critical behaviour is observed, but only to a very good approximation. The conjectured [2] superuniversality therefore holds in a rigorous fashion only when Ising variables are involved. Details of the present investigation will be reported elsewhere.

The authors are most grateful to the INFN in Pavia and to the International School for Advanced Studies in Trieste, where part of this work was carried out, for support, hospitality and the use of its facilities. This work was also supported in part by the Russian Foundation for Basic Research Grant No 96-02-16858
[1] I. F. Lyuksyutov, A. G. Naumovets and V. L. Pokrovsky, Two-Dimensional Crystals (Academic Press, London 1992).

[2] S. Chen, A. M. Farrenberg and D. P. Landau, Phys. Rev. Lett. 69, 1213 (1992); L. Schwenger, K. Budde, C. Voges and H. Pfnür, Phys. Rev. Lett. 73, 296 (1994); M. Kardar, A. L. Stella, G. Sartoni and B. Derrida, Phys. Rev. E 52, R1269 (1995).

[3] A. Aizenman and J. Wehr,Phys. Rev. Lett. 62, 2503 (1989); K. Hui and A. N. Berker, Phys. Rev. Lett. 62, 2507 (1989).

[4] R. J. Baxter, Exactly Solved Models in Statistical Mechanics (Academic Press, London 1982).

[5] I. F. Lyuksyutov and A. G. Fedorus, Sov. Phys. JETP 53, 1317 (1981); J. F. Wendelken and G. C. Wang, Phys. Rev. B 32, 7542 (1985); K. Grzelakowsky, I. F. Lyuksyutov and E. Bauer, Phys. Rev. Lett. 64, 32 (1990).

[6] M. P. M. den Nijs in Phase Transitions in Surface Films II, edited by H. Taub et al. (Plenum, New York 1991); G.Jug, Phil. Mag. B 73, 555 (1996); and references therein.

[7] Vl. S. Dotsenko and Vik. S. Dotsenko, J. Phys. A 17, L301 (1984); Vik. S. Dotsenko, J. Phys. A 18, L241
(1985).

[8] B. N. Shalaev, Phys. Rep. 237, 129 (1994); G. Jug, and B. N. Shalaev, Phys. Rev. B 543442 (1996).

[9] G. Grest and M. Widom, Phys. Rev. B 24, 6508 (1981).

[10] J.-C. Toledano, L. Michel, P. Toledano and E. Brézin, Phys. Rev. B 31, 7171 (1985), and references therein.

[11] A. B. Harris and T. C. Lubensky, Phys. Rev. Lett. 33, 1540 (1974); D. E. Khmelnitskii, Sov. Phys. JETP 68, $1960(1975)$.

[12] A. Aharony, Phys. Rev. Lett. 31, 1494 (1973).

[13] R. Shankar, Phys. Rev. Lett. 55, 453 (1985).

[14] A. D. Bruce and A. Aharony, Phys. Rev. B 11, 478 (1975); I. F. Lyuksyutov, V. L. Pokrovsky and D. E. Khmelnitskii, Sov. Phys. JETP 69, 1817 (1975); P. Bak, Phys. Rev. B 14, 3980 (1976); and references therein.

[15] A. W. W. Ludwig, Nucl. Phys. B 285, 97 (1987).

[16] Vl. S. Dotsenko, M. Picco and P. Pujol, Phys. Lett. B 377, 113 (1995); P. Pujol, Europhys. Lett. 35283 (1996).

[17] N. A. Kivel, A. S. Stepanenko and A. N. Vasil'iev, Nucl. Phys. B 424, 619 (1994).

[18] M. E. Fisher, Phys. Rev. 176, 257 (1968). 